\title{
1 Fast and Accurate Data Collection for Macromolecular Crystallography Using the
}

\section{JUNGFRAU Detector}

3 Filip Leonarski ${ }^{1}$, Sophie Redford ${ }^{1}$, Aldo Mozzanica ${ }^{1}$, Carlos Lopez-Cuenca ${ }^{1}$, Ezequiel

4 Panepucci $^{1}$, Karol Nass ${ }^{1}$, Dmitry Ozerov ${ }^{1}$, Laura Vera ${ }^{1}$, Vincent Olieric ${ }^{1}$, Dominik

5 Buntschu $^{1}$, Roman Schneider ${ }^{1}$, Gemma Tinti $^{1}$, Erik Froejdh ${ }^{1}$, Kay Diederichs ${ }^{2}$, Oliver Bunk ${ }^{1}$,

6 Bernd Schmitt ${ }^{1 *}$, Meitian Wang ${ }^{1 *}$

7

$8 \quad{ }^{1}$ Swiss Light Source, Paul Scherrer Institute, Villigen, Switzerland.

$9 \quad{ }^{2}$ Fachbereich Biologie, Universität Konstanz, Konstanz, Germany

10

$11 *$ Correspondence should be addressed to B.S. and M.W. (email: bernd.schmitt@psi.ch;

12 meitian.wang@psi.ch)

13

14

This document is the accepted manuscript version of the following article:

Leonarski, F., Redford, S., Mozzanica, A., Lopez-Cuenca, C., Panepucci, E., Nass, K., ... Wang, M. (2018). Fast and accurate data collection for macromolecular crystallography using the

JUNGFRAU detector. Nature Methods, 15(10), 799-804. https://doi.org/10.1038/s41592-018-0143-7 


\section{Abstract}

16 The accuracy of X-ray diffraction data is directly related to how the X-ray detector records

17 photons. Here we describe the application of a direct detection charge integrating pixel-array

18 detector (JUNGFRAU) in macromolecular crystallography (MX). JUNGFRAU features a

19 uniform response on the subpixel level, linear behavior toward high photon rates, and low-

20 noise performance across the whole dynamic range. These features enable accurate data to be

21 recorded at unprecedented speed. Improvements over previous generation detectors in terms

22 of data quality are demonstrated using one of the most challenging phasing methods in MX

23 with both test and real-life examples. We conclude that the adoption of the JUNGFRAU

24 detector would significantly improve the current performance of synchrotron MX beamlines

25 and make them ready for the future synchrotron light sources.

27 Keywords: Integrating detector, photon-counting detector, charge-sharing, count-rate,

28 macromolecular crystallography, diffraction data collection, data quality, native-SAD

29 phasing. 
31 Macromolecular crystallography (MX) reveals 3D structures and elucidates functions of

32 biomolecules with atomic resolution, which has enabled fundamental contributions to

33 molecular biology and structure based drug discovery ${ }^{1}$. Synchrotron radiation, together with

34 large format $2 \mathrm{D}$ detectors have been essential to the success of modern $\mathrm{MX}^{2,3}$. In parallel

35 with the evolution of synchrotron sources, several generations of X-ray detectors have been

36 developed, namely image plate (IP) ${ }^{4}$, multiwire proportional counter (MWPC) ${ }^{5}, \mathrm{X}$-ray

37 television detector (TV $)^{6}$, charge-coupled device $(\mathrm{CCD})^{7}$, and hybrid (pixel-array) photon-

38 counting detector (HPC $)^{8}$. Each generation excelled the previous one in various ways and has

39 made its marked impact on the development of MX techniques. Currently, most MX

40 beamlines are equipped with HPC detectors or have plans to do so.

41 New generations of X-ray detectors transformed MX data collection strategies as well. The

42 traditional high-dose and coarse-phi slicing data collection strategy adapted for CCD

43 detectors ${ }^{9,10}$ has been replaced by the continuous, low-dose and fine-phi slicing strategy

44 taking full advantage of HPC detectors ${ }^{11,12}$. Very recently, the EIGER detector ${ }^{13,14}$ allowed

45 including additional data collection protocols in the standard toolbox of state-of-the-art MX

46 beamlines by enabling fast raster scanning ${ }^{15}$ and serial crystallography ${ }^{16}$.

47 Among key features of HPC detectors that enabled these previously impossible data

48 collection protocols, very low noise detection and a point-spread response of a single pixel

49 are achieved by counting an incoming photon only in the pixel where it deposits at least $50 \%$

50 of its energy. Thus, photon counters have a negligible readout noise, meaning that the

51 accuracy with which they can make a measurement is limited by calibration, systematic

52 effects and Poisson statistics.

53 However, there are two intrinsic effects that may lead to photons not being detected by

54 photon-counting devices, namely charge-sharing and pile-up. Charge-sharing results in

55 photon-induced charges spreading into adjacent pixels when photons hit the sensor near to 
56 the border of pixels ("corner effect"). In such situations, the detection (counting) of the

57 photons strongly depends on the threshold settings. The calibration of the threshold becomes

58 less accurate at low photon energies $(\leq 8 \mathrm{keV})$ and a $50 \%$ threshold may not be achievable.

59 Therefore, the effects could be detrimental in low energy applications such as native-SAD

60 phasing where the most accurate measurement of intensity is needed. The effect could be

61 mitigated to a certain degree by increasing the pixel size (e.g. $170 \mu \mathrm{m}$ square in the

62 PILATUS $^{11}$ ) and by a charge summing and allocation method as implemented in

63 MEDIPIX $3^{17}$, respectively. But such measures reduce spatial resolution and count-rate

64 capability.

65 Pile-up effects occur at high photon rates due to the dead time in the readout electronic

66 circuit, which needs some time to reset before the next photon can be detected. This count-

67 rate dependence of HPC detectors leads to a non-linear response to photon flux and a count-

68 rate correction needs to be applied ${ }^{18}$. A recent development in retriggering technology ${ }^{19}$

69 extends the count-rate capacity of HPC detectors, but does not eliminate the problem. As an

70 added complication, the count-rate correction in its simplest form is only valid with a

71 constant flux of photons. However, in practice no count rate correction is applied for a

72 changing photon rate when a sharp Bragg peak moves through the diffraction condition

73 during a single exposure. These count-rate related issues are usually avoided in MX

74 measurements as these are carried out with an attenuated beam at a low rotation speed.

75 However, the count-rate capability will become acute for the next generation synchrotrons

76 with higher brilliance ${ }^{20,21}$.

77 To further develop detectors for MX, one challenge is how to overcome the aforementioned

78 charge sharing and pile-up effects while maintaining low-noise performance to the single

79 photon level. and a high dynamic range. New developments of charge integrating hybrid

80 pixel detectors could meet such challenge ${ }^{22,23} ;$ JUNGFRAU is one of them $^{24}$. Initially 
81 developed for XFEL applications ${ }^{25}$, JUNGFRAU features direct detection and a dynamic

82 gain switching technology ${ }^{24}$. Instead of counting individual photons by using a threshold,

83 JUNGFRAU measures the total amount of charge accumulated during the integration time,

84 by which it eliminates both the charge-sharing problem and the count-rate limitation entirely.

85 The three separate gains, each for a given charge range, provide both single-photon

86 sensitivity and a high dynamic range, currently limited by the $1.1 \mathrm{kHz}$ frame rate to $12 \mathrm{Mcps}$

$87 \mathrm{pxl}^{-1}$ at $12.0 \mathrm{keV} .^{26}$ The gain is switched automatically and independently per pixel

88 depending on the detected charge. The result of this approach is the combination of a linear

89 response up to much higher photon rates and noise well below the limits set by Poisson

90 counting statistics. This is illustrated in one recorded Bragg peak from a lysozyme crystal

91 (Fig. 1). In this study, we demonstrate that JUNGFRAU maintains the advantages of HPC

92 detectors for routine MX applications and offers significant improvements for emerging MX

93 applications in low-energy phasing and serial crystallography, and for future diffraction

94 limited storage rings (DLSRs). ${ }^{20}$

95

96 Results

97 JUNGFRAU maintains low-noise performance. The reliable detection of high-resolution

98 weak reflections is the foremost requirement for X-ray detectors in MX. In this aspect, HPC

99 detectors are near to ideal because of their very low noise detection with single-photon

100 sensitivity and single-pixel point-spread function. To analyze JUNGFRAU's low-noise

101 performance, we compared it with EIGER, a widely used HPC detector, in the most common

102 MX application - native data collection with $12.4 \mathrm{keV}$ X-rays. These two detectors are

103 particularly suited to perform a comparison of photon counting versus charge integrating

104 methods since they have the same pixel size, same sensor area, same hybrid nature, and

105 similar sensor thickness. Two data sets were collected with the same thaumatin crystal under 
106 identical X-ray beam conditions (Online Methods) - one with a JUNGFRAU 1Mpixel

107 detector (JF1M; Supplementary Fig. 1) and the other with an EIGER 1Mpixel detector

108 (E1M). The dose was set very low $(0.6 \mathrm{kGy} /$ data set $)$ deliberately to have Poisson statistics

109 limited noises for the whole resolution range.

110 The two data sets have almost the same quality with similar $R_{\text {meas }}$ (Fig. 2a, Supplementary

111 Table 1 and 2). Both detectors recorded very weak intensities down to the one photon level at

112 resolutions of $2 \AA$ and higher. The half-data set correlations $\left(C C_{1 / 2}\right)$, intensities, and $I / \sigma$ are

113 marginally lower for JF1M because of its slightly reduced duty cycle and thinner Si sensor

114 (Fig. 2b,c,d). After normalizing for detector duty cycle (JF1M 95\% vs. E1M 99.7\%) and

115 sensor thickness (JF1M $320 \mu \mathrm{m} v s . \mathrm{E} 1 \mathrm{M} 450 \mu \mathrm{m})($ Online Methods), the intensities and $I / \sigma$

116 values are virtually the same in the whole resolution range for both detectors (Fig. 2c,d). As

117 the maximum duty cycle of JUNGFRAU will be improved to the $99 \%$ level in the future, and

118 since thicker sensors may be chosen during detector construction, it is expected that the

119 performance of JUNGFRAU will approach the excellent results of EIGER for weak

120 diffraction.

121 JUNGFRAU enables data collection with full flux. To test JUNGFRAU for high count-rate

122 applications, we performed a series of experiments with increasing flux (beam transmission

$1231,20,50,100 \%)$ and rotation speed $(1,20,50$, and $100 \%$ s) from a thaumatin crystal at $6 \mathrm{keV}$

124 (Online Methods). Compare with the previously published similar experiments with an E1M

125 detector, where the data quality gradually deteriorated with increased flux due to count-rate

$126 \operatorname{limit}^{19}$, the four JF1M data sets were of very similar quality as judged by $R_{\text {meas }}$ and $I / \sigma$ (Fig.

127 3a,b, Supplementary Table 1and 3) and their intensity correlations are in excellent agreement

128 across the whole dynamic range (Fig. 3c). In the JF1M $100^{\circ} / \mathrm{s}$ data set, the top 1 and 6

129 percentile of strong reflections contained data recorded with photon rates of 500 and 200

$130 \mathrm{Mcps} \mathrm{mm}^{-2}$, respectively (Online Methods, Supplementary Table 4), which are beyond or 
131 close to the count-rate limit of typical HPC detectors respectively. The strongest reflection in

132 the dataset $(h=1, k=1, l=1)$ shows that JUNGFRAU operated at $1.136 \mathrm{kHz}$ is capable of

133 measuring photon rates of more than 4,300 $\mathrm{Mcps} \mathrm{mm}^{-2}$ at an X-ray energy of $6 \mathrm{keV}$ (Fig. 3d).

134 JUNGFRAU improves data accuracy. A native-SAD phasing experiment was chosen to

135 assess the quality of data obtained with the JUNGFRAU detector because this method relies

136 on very accurate measurements of reflection intensities to derive phase ${ }^{27,28}$. A thaumatin

137 crystal was measured with $6 \mathrm{keV}$ X-rays using both the JF1M and E1M detectors (Online

138 Methods). Two settings were used for the E1M - one with the default 50\% threshold (E1M-

$13950)$, and the other with $60 \%(\mathrm{E} 1 \mathrm{M}-60)$ to simulate a situation where the lowest possible

140 threshold is higher than $50 \%$ of the photon energy $(<6 \mathrm{keV})$. For the direct comparison, all

141 measurements were made at the same position of the same crystal with identical data

142 collection parameters (Online Methods). For this thaumatin crystal, the typical size of a

143 diffraction spot is few pixels on average and is smaller at low resolution than at high

144 resolution due to the parallax in the diffraction geometry (Supplementary Fig. 2).

145 The recorded JF1M data are of high quality as evaluated by $R_{\text {meas }}$ (Fig. 4a, Supplementary

146 Table 1 and 5) and $I / \sigma$ (Supplementary Fig. 3). The $R_{\text {meas }}$ of $2.5 \%$ at the lowest resolution

147 shell reflects the excellent consistency between individual measurements. The $R_{\text {meas }}$ gradually

148 increases with the resolution to $5 \%$ at $2.7 \AA$ with a characteristic bump around $6 \AA$ due to an

149 intensity distribution typical to most protein crystals. In contrast, the E1M-50 data are

150 noticeably worse at low resolution with $R_{\text {meas }}$ of $5 \%$. The data quality further deteriorates in

151 the E1M-60 data. Such differences have a significant impact on the average density in the

152 anomalous difference Fourier map for sulfur atoms $\left(<\mathrm{S}_{\mathrm{anom}}>\right.$, Fig. $\left.4 \mathrm{~b}\right)$. The $<\mathrm{S}_{\mathrm{anom}}>$ is a

153 useful metric for structure solvability in SAD phasing, and a value above $10 \sigma$ usually

154 indicates sufficient signal for structure solution ${ }^{29}$. The $<\mathrm{S}_{\text {anom }}>$ values were $10.2 \sigma, 8.9 \sigma$, and

$1558.3 \sigma$ for $75^{\circ}$ using JF1M, E1M-50, and E1M-60, respectively. Two to three times more data 
156 were required to elevate $<\mathrm{S}_{\text {anom }}>$ above $10 \sigma$ for E1M data (Fig. $4 b$ ). Indeed, the sub-structure

157 was solved using SHELXC/D ${ }^{30}$ with merely $60^{\circ} \mathrm{JF} 1 \mathrm{M}$ data (Fig. $4 \mathrm{c}$ ) while the same $60^{\circ}$ data

158 from E1M-50 failed to produce a structure solution (Fig. 4d).

159 To understand the origin of the above discrepancy in data quality obtained with JF1M and

160 E1M detectors, we quantified the uniform response in the sub-pixel level by mapping the

161 deviation of intensities in fractional coordinates of one pixel based on the refined position of

162 reflections $\left(\Delta_{x, y}\right)$. Then an average pixel map was calculated with the normalized $\Delta_{x, y}$

163 (Online Methods; Eq. 4). In the case of JF1M the pixel map is essentially featureless,

164 indicating no significant bias in intensity measurement regardless of where the reflection was

165 located within the pixel (Fig. 5a), as expected for a charge integrating detector. However, in

166 the case of E1M-50 there is a systematic difference between reflections centered in the

167 middle of a pixel and near to the corners (Fig. 5b), and the magnitude of the effect increases

168 with the detector threshold (Fig. 5c). Since most diffraction spots of the crystal are elongated

169 in the vertical direction (Supplementary Fig. 2), the effect is much stronger in the horizontal

170 direction in the E1M pixel maps. The non-uniformity in EIGER is likely to be attributed to

171 the corner effect, inaccuracy in threshold calibration and count-rate corrections at low energy.

172 To estimate the contribution of these effects on crystallographic $R_{m e a s}, R_{p x l}$ was introduced as

173 a measure of systematic errors caused by the non-uniformity across pixels by averaging out

174 random errors (Online Methods; Eq. 5). $R_{p x l}$ values are below 1\% for JF1M (Fig. 5d). For

175 E1M data, $R_{p x l}$ has a resolution dependent behavior because the detector non-uniformity is

176 more visible for sharp low-resolution spots. It rises gradually from $3 \AA$ towards lower

177 resolution and becomes a main contributor to the higher $R_{\text {meas }}$ in the low-resolution range

178 (Fig. 5e,f).

179 The sub-pixel and inter-pixel uniformity in JF1M was also verified by detector shifting

180 experiments, in which we measured data sets with JF1M shifted by one third and two thirds 
181 of a pixel in a diagonal direction orthogonal to the beam direction. By combining two data

182 sets - one with and one without the JF1M shift, the data accuracy is the same as measured by

$183<\mathrm{S}_{\mathrm{anom}}>$, compared to the same amount of data collected with only one detector position (Fig.

$1845 \mathrm{~g})$. In the similar detector shift experiments with E1M, the data accuracy was significantly

185 improved by combining data from two detector positions to average out the non-uniform

186 response within and between pixels in E1M (Fig. 5h,i). This analysis confirms that

187 JUNGFRAU has good uniform responses within pixels, which permits the measurement of

188 reflection intensities with high accuracy even at low X-ray energies and with diffraction peak

189 sizes comparable to the size of the pixel.

190 JUNGFRAU expedites experimental phasing. Accurate measurement of reflection

191 intensities with high incoming photon rates, made possible by JUNGFRAU, enables efficient

192 usage of the full flux provided by an undulator beamline efficiently for experimental phasing

193 with anomalous diffraction, whose success stringently depends on the data accuracy. We

194 have chosen one of the most challenging phasing methods - native-SAD to demonstrate

195 JUNGFRAU's distinct advantages.

196 First, we show that a flash of low-energy X-rays of less than a second is sufficient for native-

197 SAD phasing using a thaumatin crystal as the model system. A total of $60^{\circ}$ of data were

198 collected from one crystal at $6 \mathrm{keV}$ with a rotation speed of $100^{\circ} / \mathrm{s}$. The entire exposure lasted

199 for 0.6 seconds. With this data, all sulfurs were identified readily with SHELXD ${ }^{30}$ and the

200 resulting electron density map from CRANK2 ${ }^{31}$ was of excellent quality (Fig. 6a). The

201 multiplicity of the data set was only 2.1 (Supplementary Table 1 and 6). This serves as

202 another testament that the uniform response of JUNGFRAU allows achieving high data

203 accuracy with minimum averaging.

204 To probe the limit further, we attempted a native-SAD experiment with $12.4 \mathrm{keV}$ X-rays

205 using a lysozyme crystal. This energy is unfavorable for native-SAD experiment because the 
anomalous scattering factor $-f^{\prime \prime}$ of sulfur is only $0.24 \mathrm{e}^{-}$. Although the estimated average differences in structure factors of Friedel pairs (i.e., Bijvoet ratio) was as low as $0.6 \%$, the structure was phased by SHELXC/D/E with a $500^{\circ}$ data set measured in only five seconds (Fig. 6b). In this case, high-resolution data up to $1.15 \AA$ also helped in accumulating weak but significant anomalous signals (Supplementary Table 1 and 6).

211 Next, we selected the $E$. coli aminopeptidase N (PepN) representing a real-life example.

212 PepN is a $101 \mathrm{kDa}$ protein (891 residues) containing 30 sulfurs, 1 bromine and 1 zinc atom.

213 Because the average diffraction power of PepN is much weaker compared to test proteins like 214 thaumatin and lysozyme, the rotation speed of data collection was limited to $10 \%$ s to ensure

215 sufficient exposure per diffraction image with a flux of $2.7 \times 10^{11} \mathrm{ph} / \mathrm{s}$ at $6 \mathrm{keV}$. A $600^{\circ}$ data

216 set, collected in one minute (Supplementary Table 1 and 6), allowed a straightforward

217 structure solution using SHELXC/D/E (Fig. 6c). If five to ten times more flux would be

218 available, we expect that the same structure could be solved within 5-10 seconds using 50-

$219100 \%$ s rotation.

\section{Discussion}

222 The diffraction pattern of a macromolecular crystal contains thousands of sharp Bragg peaks 223 with large variations in their intensities. Solving structures, especially by experimental 224 phasing methods, requires accurate measurement of strong Bragg peaks and the atomic 225 features of a structure are determined by precise recording of weak Bragg peaks at high resolution. Measuring strong and weak intensities has different challenges. Ideally a detector

227 should have uniform response across a large surface area on both the pixel and sub-pixel

228 level, high dynamic range with linear response, high sensitivity to the single photon level, 229 single-pixel point spread function and continuous readout. None of the previous generations 230 of detectors had addressed all these requirements satisfactorily. In response to this challenge, 
231 JUNGFRAU uses a charge integrating readout chip and the successful direct detection hybrid 232 pixel detector technology to provide a low noise performance over the whole dynamic range 233 of $10^{4} 12 \mathrm{keV}$ photons per frame per pixel and a uniform response within and across pixels.

234 From a data accuracy and precision point of view, the requirements are most stringent in 235 experimental phasing because the small anomalous differences $(\leq 1 \%)$ between Bragg peaks 236 related by Friedel's law and crystal symmetry lead to reliable evaluation of phases. If the size 237 of Bragg spots is comparable to or smaller than the pixel size of the detector, their 238 measurement accuracy will be compromised when measured with detectors with non-uniform 239 sub-pixel response such as HPCs. The smaller the spot is in comparison to the detector pixel 240 size, the more severe the effect will be. In practice, this shortcoming is mitigated 241 conveniently by collecting true high-multiplicity data using a multi-axis goniometer ${ }^{28}$, but at 242 a cost of increasing the X-ray dose and thus the radiation damage ${ }^{32}$ and experiment time. In 243 this work, we have demonstrated that JUNGFRAU permits an accurate measurement of 244 photons independent of where they land on the detector surface, which allows obtaining 245 highly accurate data, hence achieving experimental phasing with minimum X-ray dose and 246 reduced multiplicity (Fig. 4). Therefore, the JUNGFRAU detector holds great promise, 247 especially for native-SAD phasing using X-rays in the 3-5 keV range where the calibration of 248 HPC detectors is particularly challenging. Furthermore, the measurements can be carried out 249 faster with high flux because JUNGFRAU is not count-rate limited. This unique combination 250 of accuracy and speed was demonstrated using native-SAD experiments (Fig. 6a,b) with a 251 rotation speed of $100^{\circ} / \mathrm{s}$, a speed that was considered of no practical use in the past, but now 252 can be exploited to develop novel data collection strategies.

253 Time-resolved crystallography with Laue methods was made possible by 3rd generation 254 high-energy synchrotron facilities ${ }^{33}$. However, the Laue methods required large crystals and 255 specialized beamlines. Very recently, the emerging serial synchrotron crystallography (SSX) 
256 has broadened horizons of time-resolved crystallography by introducing novel crystal

257 delivery techniques and automated data collection methods with fast frame-rate detectors ${ }^{34-37}$.

258 To further improve the efficiency of SSX methods and the time-resolution, the available flux

259 density can be increased 100-1000 fold by using wide-bandpass X-rays ${ }^{38}$. Then integrating

260 detectors become indispensable. JUNGFRAU technology meets the challenges nicely and

261 will allow the study of biologically relevant dynamics down to microsecond timescales in a

262 pump-probe fashion at synchrotrons.

263 There are challenges in implementing JUNGFRAU at MX beamlines. Dark runs (i.e. without

264 X-rays) are required to calculate pedestals for each gain and need to be included in the data

265 acquisition sequence with minimum overhead. The raw data need to be corrected and

266 converted to photons before the data volume can be reduced by frame summation. This

267 requires handling high data rates $(4 \mathrm{~GB} / \mathrm{s}$ per $1 \mathrm{M}$ pixels) for real time data analysis. The

268 solutions are in active development at the PSI to match the robustness and simplicity of

269 operating HPC detectors. Such challenges are essentially the same for XFEL serial

270 crystallography applications ${ }^{39-41}$.

271 Using common data acquisition protocols and experimental conditions in terms of photon

272 energy, count-rate and sharpness of the diffraction spots, the HPC detector produces data of

273 high quality for the majority of MX applications currently. But pushing the frontiers of

274 method and technology development further, even this latest detector generation has its

275 limitations. In these scenarios, the improvement in data accuracy and data collection speed

276 obtained with the JF1M detector used here is remarkable. The ultimate obtainable data

277 quality from a given crystal depends on many factors, but it is evident that detectors like

278 JUNGFRAU will be pivotal in getting close to this limit. This is a major advancement in the

279 continuous quest for improving MX data quality and will prompt development of low-noise 
280 instruments in the next generation MX beamlines to capitalize on the full potential of the next

281 generation synchrotron sources like DLSRs in the coming decade.

282

283 Acknowledgements

284 We thank C. Tarnus, C. Schmitt and S. Albrecht for the preparation of PepN crystals.

285

286 Author contributions

287 M.W., O.B. and B.S. conceived the research; S.R., A.M., C.L.-C. built and calibrated JF1M

288 detector; A.M., D.B., and R.S. installed JF1M and E1M detectors at beamlines X06SA; F.L.,

289 A.M. and E.P. developed diffraction data collection software; L.V. and V.O. prepared

290 samples; F.L., S.R., A.M., E.P., and M.W. collected data; F.L., S.R., K.N., D.O., G.T., E.F.,

291 K.D., and M.W. analyzed data; F.L., S.R., O.B, and M.W. wrote the manuscript with

292 contributions from all authors.

293

294 Competing financial interests

295 The authors declare no competing financial interests.

\section{References}

297

298

299

300

301

302

303

304

305

306

307

308
1 Jaskolski, M., Dauter, Z. \& Wlodawer, A. A brief history of macromolecular crystallography, illustrated by a family tree and its Nobel fruits. FEBS Journal 281, 3985-4009, doi:10.1111/febs.12796 (2014).

2 Duke, E. M. H. \& Johnson, L. N. Macromolecular crystallography at synchrotron radiation sources: current status and future developments. Proceedings of the Royal Society A: Mathematical, Physical and Engineering Sciences 466, 3421-3452, doi:10.1098/rspa.2010.0448 (2010).

3 Gruner, S. M., Eikenberry, E. F. \& Tate, M. W. in International Tables for Crystallography Vol. F Ch. 7.1, 143-147 (2006).

4 Seggern, H. v. Photostimulable x-ray storage phosphors: a review of present understanding. Brazilian Journal of Physics 29, 254-268, doi:10.1590/s010397331999000200008 (1999). 
3095 Howard, A. J. et al. The use of an imaging proportional counter in macromolecular

6 Arndt, U. W. X-ray television area detectors. Synchrotron Radiation News 3, 17-22, doi:10.1080/08940889008602568 (1990).

7 Gruner, S. M., Tate, M. W. \& Eikenberry, E. F. Charge-coupled device area X-ray detectors. Rev Sci Instrum 73, 2815-2842, doi:10.1063/1.1488674 (2002).

8 Graafsma, H. in Semiconductor Radiation Detection Systems (ed Krzysztof Iniewski) Ch. 10, 217-236 (CRC Press, 2010).

9 Waterman, D. \& Evans, G. Estimation of errors in diffraction data measured by CCD area detectors. Journal of Applied Crystallography 43, 1356-1371, doi:10.1107/s0021889810033418 (2010).

10 Dauter, Z. Data-collection strategies. Acta Crystallographica Section D Biological Crystallography 55, 1703-1717, doi:10.1107/s0907444999008367 (1999).

11 Broennimann, C. et al. The PILATUS 1M detector. J Synchrotron Radiat 13, 120 130, doi:10.1107/s0909049505038665 (2006).

12 Mueller, M., Wang, M. \& Schulze-Briese, C. Optimal fine phi-slicing for singlephoton-counting pixel detectors. Acta Cryst 68, D42-56, doi:10.1107/S0907444911049833 (2012).

13 Dinapoli, R. et al. EIGER: Next generation single photon counting detector for X-ray applications. Nuclear Instruments and Methods in Physics Research Section A: Accelerators, Spectrometers, Detectors and Associated Equipment 650, 79-83, doi:10.1016/j.nima.2010.12.005 (2011).

14 Casanas, A. et al. EIGER detector: application in macromolecular crystallography. Acta Cryst D72, 1036-1048, doi:10.1107/S2059798316012304 (2016).

15 Wojdyla, J. A. et al. Fast two-dimensional grid and transmission X-ray microscopy scanning methods for visualizing and characterizing protein crystals. Journal of Applied Crystallography 49, 944-952, doi:10.1107/s1600576716006233 (2016).

16 Diederichs, K. \& Wang, M. in Protein Crystallography Methods in Molecular Biology Ch. 10, 239-272 (2017).

17 Ballabriga, R. et al. The Medipix3RX: a high resolution, zero dead-time pixel detector readout chip allowing spectroscopic imaging. J Instrum 8, C02016-C02016, doi:10.1088/1748-0221/8/02/c02016 (2013).

18 Sobott, B. A. et al. Success and failure of dead-time models as applied to hybrid pixel detectors in high-flux applications. J Synchrotron Radiat 20, 347-354, doi:10.1107/s0909049513000411 (2013).

19 Loeliger, T. et al. in 2012 IEEE Nuclear Science Symposium and Medical Imaging Conference Record (NSS/MIC) 610-615 (2012).

20 Eriksson, M., van der Veen, J. F. \& Quitmann, C. Diffraction-limited storage rings - a window to the science of tomorrow. J Synchrotron Radiat 21, 837-842, doi:10.1107/s 1600577514019286 (2014).

21 Denes, P. \& Schmitt, B. Pixel detectors for diffraction-limited storage rings. $J$ Synchrotron Radiat 21, 1006-1010, doi:10.1107/s1600577514017135 (2014).

22 Graafsma, H., Becker, J. \& Gruner, S. M. in Synchrotron Light Sources and FreeElectron Lasers (eds E. Jaeschke, S. Khan, J. R. Schneider, \& J.B. Hastings) Ch. 37, 1029-1054 (Springer International Publishing, 2016).

23 Tate, M. W. et al. A Medium-Format, Mixed-Mode Pixel Array Detector for Kilohertz X-ray Imaging. Journal of Physics: Conference Series 425, doi:10.1088/1742-6596/425/6/062004 (2013). 
24 Mozzanica, A. et al. Characterization results of the JUNGFRAU full scale readout ASIC. J Instrum 11, C02047-C02047, doi:10.1088/1748-0221/11/02/c02047 (2016).

25 Chapman, H. N. et al. Femtosecond X-ray protein nanocrystallography. Nature 470, 73-77, doi:10.1038/nature09750 (2011).

27 Liu, Q. et al. Structures from Anomalous Diffraction of Native Biological Macromolecules. Science 336, 1033-1037, doi:10.1126/science.1218753 (2012).

28 Weinert, T. et al. Fast native-SAD phasing for routine macromolecular structure determination. Nat Methods 12, 131-U163, doi:10.1038/nmeth.3211 (2015).

29 Terwilliger, T. C. et al. Can I solve my structure by SAD phasing? Anomalous signal in SAD phasing. Acta Crystallographica Section D Structural Biology 72, 346-358, doi:10.1107/s2059798315019269 (2016).

30 Sheldrick, G. M. Experimental phasing with SHELXC/D/E: combining chain tracing with density modification. Acta Cryst D66, 479-485, doi:10.1107/s0907444909038360 (2010).

31 Skubák, P. \& Pannu, N. S. Automatic protein structure solution from weak X-ray data. Nature Communications 4, doi:10.1038/ncomms3777 (2013).

32 Garman, E. F. Radiation damage in macromolecular crystallography: what is it and why should we care? Acta Crystallographica Section D Biological Crystallography 66, 339-351, doi:10.1107/s0907444910008656 (2010).

33 Neutze, R. \& Moffat, K. Time-resolved structural studies at synchrotrons and X-ray free electron lasers: opportunities and challenges. Current Opinion in Structural Biology 22, 651-659, doi:10.1016/j.sbi.2012.08.006 (2012).

34 Gati, C. et al. Serial crystallography on in vivo grown microcrystals using synchrotron radiation. IUCrJ 1, 87-94, doi:10.1107/s2052252513033939 (2014).

35 Weinert, T. et al. Serial millisecond crystallography for routine room-temperature structure determination at synchrotrons. Nature Communications $\mathbf{8}$, doi:10.1038/s41467-017-00630-4 (2017).

36 Beyerlein, K. R. et al. Mix-and-diffuse serial synchrotron crystallography. IUCrJ 4, 769-777, doi:10.1107/s2052252517013124 (2017).

37 Gruner, S. M. \& Lattman, E. E. Biostructural Science Inspired by Next-Generation XRay Sources. Annual Review of Biophysics 44, 33-51, doi:10.1146/annurev-biophys060414-033813 (2015).

38 Meents, A. et al. Pink-beam serial crystallography. Nature Communications 8, doi:10.1038/s41467-017-01417-3 (2017).

39 Barty, A. et al. Cheetah: software for high-throughput reduction and analysis of serial femtosecond X-ray diffraction data. Journal of Applied Crystallography 47, 11181131, doi:10.1107/s1600576714007626 (2014).

40 Mariani, V. et al. OnDA: online data analysis and feedback for serial X-ray imaging. Journal of Applied Crystallography 49, 1073-1080, doi:10.1107/s1600576716007469 (2016).

41 Zeldin, O. B. et al. Data Exploration Toolkitfor serial diffraction experiments. Acta Crystallographica Section D Biological Crystallography 71, 352-356, doi:10.1107/s1399004714025875 (2015). 
406 Figures legends

407 Figure 1 Demonstration of the dynamic gain switching of the JUNGFRAU detector. (Left) A

408 diffraction image from a lysozyme crystal measured without beam attenuation at X06SA

409 beamline, SLS was shown. (Middle) Zoom-in on a Bragg peak showing the number of

410 photons detected, where the central pixel was measured in low gain (red), tails of the peak

411 were measured in medium gain (yellow), while the background is measured in high gain

412 (blue). (Right) The relationship between a charge integrated by the pixel and its ADC count

413 output for three gains (high (G0), medium (G1) and low (G2)).

414 Figure 2 Data quality comparison between JF1M and E1M for routine MX applications. All

415 comparisons are made as a function of resolution. (A) $R_{\text {meas }}$ values. (B) $C C_{1 / 2}$ values. (C) The

416 intensity $\left(<>_{\text {unmrgd }}\right)$ values with and without normalization for the duty cycle and sensor

417 thickness. The $<I>{ }_{\text {unmrgd }}$ values are prior to application of Lorentz and polarization

418 corrections. (D) $<I / \sigma>$ unmrgd values.

419 Figure 3 Comparison of measurements with different photon rates using the JUNGFRAU 420 detector. (A,B) The $R_{\text {meas }}$ and $\langle I / \sigma\rangle_{\operatorname{mrgd}}$ values for measurements obtained with beam 421 transmissions at $1 \%, 20 \%, 50 \%$, and $100 \%$. (C) The correlation of integrated intensities of 422 reflections measured with beam transmission of $100 \%$ with respective intensities measured at $4231 \%$ transmission (Pearson correlation coefficient of 0.98 ). Blue dots represent reflections 424 with photon-rate $>200 \mathrm{Mcps} \mathrm{mm}^{-2}$. (D) The correlation of the estimated photon rate 425 extracted from the single pixel of a reflection with highest counts between $1 \%$ and $100 \%$ 426 transmission data sets (Pearson correlation coefficient of 0.93). The spread of the plot comes 427 from the fact that depending on the slicing position of a reflection, the number of photons 428 might differ for the pixel with the highest counts. The orange line represents an ideal linear 429 response. The black curve is the theoretical behavior of a paralyzable counter with dead time 
430 of $280 \mathrm{~ns}$ and the horizontal dotted black line marks a corresponding count-rate limit (see

431 Online Methods).

432 Figure 4 Comparison of $6 \mathrm{keV}$ data from a thaumatin crystal measured with JF1M and E1M

433 detectors (two threshold settings for E1M). (A) The crystallographic $R_{\text {meas }}$ as a function of

434 resolution. (B) The anomalous signal $\left(<\mathrm{S}_{\mathrm{anom}}>\right)$ as a function of total rotation range. The

435 magenta line represents the threshold for structure solvability. (C) SHELXD sub-structure

436 determination from 200 trials with $60^{\circ} \mathrm{JF} 1 \mathrm{M}$ data. The correct solution with high $C C_{\text {all }}$ and

$437 C C_{\text {weak }}$ is marked as a red dot. (D) SHELXD sub-structure determination from 5000 trails

438 with $60^{\circ} \mathrm{E} 1 \mathrm{M}-50$ data.

439 Figure 5 The sub-pixel uniformity characterizations of the JUNGFRAU and EIGER

440 detectors. The left column shows JF1M data, the central column shows E1M-50 data, and the

441 right column shows E1M-60 data. (A, B, C) Pixel maps for low-resolution data (> $10 \AA$ ) (Eq.

442 4). (D, E, F) $R_{p x l}$ (Eq. 5) and crystallographic $R_{\text {meas }}$ as a function of resolution. (G, H, I)

443 Measured anomalous signals from combined data sets with and without detector shifts.

444 Reflections flagged as misfit in XDS were included in calculations of the statistics.

445 Figure 6 Fast native-SAD phasing with an unattenuated beam at both $6 \mathrm{keV}$ and $12.4 \mathrm{keV}$ 446 with JF1M. For each case, results of the substructure search with SHELXD (left) - the correct 447 solutions with high $C C_{\text {all }}$ and $C C_{\text {weak }}$ are marked as red dots, and the electron density maps 448 (right) are shown. (A) Thaumatin with $60^{\circ}$ of data measured in $600 \mathrm{~ms}$ at $6 \mathrm{keV}$. Density map 449 obtained after density modification, automated tracing and refinement with CRANK2. (B) 450 Lysozyme with $500^{\circ}$ of data measured in $5 \mathrm{~s}$ at $12.4 \mathrm{keV}$. Density map obtained after density 451 modification and automated tracing with SHELXE. (C) Aminopeptidase with $600^{\circ}$ of data 452 measured in one minute at $6 \mathrm{keV}$. Density map obtained after density modification and 453 automated tracing with SHELXE. 


\section{Online Methods}

457 General experiment setup. Experiments were performed at the X06SA protein crystallography undulator beamline, Swiss Light Source, at beam energies of both $12.4 \mathrm{keV}$ and $6 \mathrm{keV}$. The beam size was adjusted to $80 \times 80 \mu \mathrm{m}^{2}$ and the flux for non-attenuated beam

460 was $1.6 \times 10^{12} \mathrm{ph} / \mathrm{s}$ and $2.7 \times 10^{11} \mathrm{ph} / \mathrm{s}$ for 12.4 and $6 \mathrm{keV}$, respectively. For $12.4 \mathrm{keV}$

461 measurements default beamline settings were used, while for $6 \mathrm{keV}$ the monochromator was 462 detuned by $0.002^{\circ}$ to remove higher harmonics. The beamstop was placed $7 \mathrm{~mm}$ from the 463 sample, which shadowed reflections with resolution lower than $10 \AA$ for $12.4 \mathrm{keV}$ X-rays.

464 The beamline was equipped with a motorized stage allowing movement of the JUNGFRAU 465 and EIGER detectors in three directions. Sample to detector distance could be changed in the $46640-120 \mathrm{~mm}$ range, while the two perpendicular directions could be set within $20 \mathrm{~mm}$ from the 467 detector center. The motor resolution was $2.5 \mu \mathrm{m}$. Crystal centering and EIGER data 468 collection were controlled using the DA+ software ${ }^{42}$. The JUNGFRAU data collection was 469 carried out with customized programs. Life Sciences Reporting Summary is available online. 470 JUNGFRAU 1M detector characteristics. The unique feature of the JUNGFRAU detector 471 is its dynamic gain switching with three gain levels accommodating both single photon 472 sensitivity and high dynamic range. The JUNGFRAU detector is modular and each module 473 has an active area of $4 \times 8 \mathrm{~cm}^{2}$ with eight application specific integrated circuits (ASIC) and 474 contains $\sim 500,000$ pixels of $75 \mu \mathrm{m}$ pitch. The sensor geometry is identical to that of EIGER. 475 Modules are independent in terms of read-out, each having a dedicated $10 \mathrm{~Gb} / \mathrm{s}$ Ethernet link 476 and can be arranged into various geometric shapes. Currently silicon of $320 \mu \mathrm{m}$ thickness is 477 used for the JUNGFRAU sensor. A thicker sensor, such as the $450 \mu \mathrm{m}$ thick sensor of 478 EIGER, could also be used. 
479 The JUNGFRAU system used in this experiment consisted of two modules, giving a one

480 million-pixel system (JF1M). The gap between the modules, insensitive to X-rays, was

481 estimated at approx. $2.7 \mathrm{~mm}$ (36 pixels). The system was operated with $880 \mu$ s frame time

$482(1,136 \mathrm{~Hz})$ and $840 \mu$ s integration time (i.e., a duty cycle of 95\%). This almost continuous

483 mode is very different from the pulse mode used for XFEL applications. The integration time

484 and the frame rate were determined by detector characteristics coupled with the desire to

485 achieve as high a duty cycle as possible. In order to limit the integration of the leakage

486 current, the present maximum frame rate of $1,136 \mathrm{~Hz}$ was used, corresponding to a period of

$487880 \mu \mathrm{s} .40 \mu \mathrm{s}$ is required between the end of integration and the start of readout, to move

488 charge through the chip. This leaves an integration time of $840 \mu \mathrm{s}$. The readout of the

489 previous frame is then performed during the integration of the next frame. To further reduce

490 the leakage current, the detector was cooled to $-12^{\circ} \mathrm{C}$. Other parameters, such as the internal

491 ASIC voltages, sensor bias voltage, timings, are standard as also used in XFELs. A dedicated

492 computer was used to control the detector and to store frames during data collection. The

493 frame rate $(1,136 \mathrm{~Hz})$ and frame size (1 million pixels in 16-bit) required a wide bandwidth

494 of $2.3 \mathrm{~GB} / \mathrm{s}$ to prevent frame loss.

495 The ASIC of JUNGFRAU is designed to keep the readout noise below Poisson statistics and 496 to have single photon sensitivity at energies as low as $2 \mathrm{keV}$. The readout noise is estimated

497 as 200 electrons for the high gain with an integration time of $840 \mu$ s. Operated at an XFEL

498 with an integration time of $10 \mu \mathrm{s}$, the noise is reduced to 70 electrons.

499 The maximum number of counts is determined by the charge range of the low gain. Since the

500 induced charge from a single photon is proportional to its energy, the dynamic range is

501 effectively doubled at $6 \mathrm{keV}$ in comparison to $12 \mathrm{keV}$. When operated in the $1.1 \mathrm{kHz}$ frame

502 rate, the dynamic range is about 12 and $25 \mathrm{Mcps} \mathrm{pxl}^{-1}\left(2100\right.$ and $\left.4400 \mathrm{Mcps} \mathrm{mm}^{-2}\right)$ at 12 and

$5036 \mathrm{keV}$, respectively and roughly doubles at the foreseen operation frame rate of $2.3 \mathrm{kHz}$. 
504 JUNGFRAU data format and image processing. The result of each JUNGFRAU

505 measurement is a raw image. For each pixel the gain level (2-bit) and digitized accumulated

506 charge (14-bit) in arbitrary detector units (ADUs) are recorded. To convert the raw signal to

507 photon energy, six constants are needed per pixel - for each of the three gain levels one needs

508 to know the amplification factor, i.e. the ratio of arbitrary detector charge units and energy,

509 and the pedestal, i.e. the offset corresponding to the pixel's dark output.

510 Gain values are assumed to be invariant of experimental conditions and have been measured

511 for the JF1M previously ${ }^{26}$. The achieved accuracy of the gain calibration is at about $1 \%$ level

512 currently ${ }^{43}$. The pedestal, on the other hand, depends strongly on experimental conditions,

513 especially on the integration time and the sensor temperature. Therefore, dedicated dark

514 measurements were performed before every data set measured with JF1M, consisting of 5000

515 frames in high gain $(1,136 \mathrm{~Hz}), 1000$ in medium gain $(500 \mathrm{~Hz})$ and 1000 in low gain $(200$

$516 \mathrm{~Hz}$ ). Since a pedestal drift was observed immediately after the detector starts recording,

517 related to the changes in temperature and operation mode for this particularly long integration

518 time, a delay of $10 \mathrm{~s}$ was introduced between detector start and shutter opening. Frames

519 measured during this period were used to dynamically track the pedestal but were not used

520 for data processing. Currently, such procedure introduces approx. 60 seconds delay for an

521 experiment. It is expected that much shorter and less frequent measurement would be

522 sufficient, and optimization for efficiency is under study.

523 Pedestal subtraction and gain conversion were applied on raw images after data collection

524 finished. The energy measured per pixel was converted to a photon count by dividing by the

525 incoming photon energy. Geometric corrections were performed to account for the $\sim 36$ pixel

526 gap between the modules, and the multi-size pixels between the ASICs. Finally an optional

527 frame summation was performed. Since result of conversion of ADU to photon counts is not

528 integers, and floating point values are not supported by common crystallography data 
529 processing packages, these values were rounded to integer and final images were saved in the

$530 \mathrm{CBF}$ format ${ }^{44}$. Pixels which saturated the highest possible ADU count at the lowest gain were 531 marked as overloads.

532 EIGER 1M detector. Comparative data were obtained with an EIGER 1M detector (E1M,

533 Dectris Ltd.). The detector consists of two modules each with $\sim 500,000$ pixels of $75 \mu \mathrm{m}$

534 pitch, i.e. the format is directly comparable to the one of the JUNGFRAU 1M detector used

535 for these experiments. The sensor thickness is $450 \mu \mathrm{m}$. Images were saved in HDF5 format.

536 Protein crystal preparation. Lysozyme was dissolved at $50 \mathrm{mg} / \mathrm{ml}$ in $50 \mathrm{mM}$, Sodium

537 Acetate $\mathrm{pH} 4.5$ and crystallized in 5\% PEG MME 5000, $2 \mathrm{M} \mathrm{NaCl}, 50 \mathrm{mM}$ Sodium Acetate

$538 \mathrm{pH} 4.5,25 \%$ ethylene glycol. Thaumatin was suspended at $50 \mathrm{mg} / \mathrm{ml}$ in water and

539 crystallized in $24 \%$ Sodium Potassium Tartrate, 100 mM Bis-Tris Propane pH 6.5.

540 Aminopeptidase PepN crystallization was carried out with inhibitor I1 according to the

541 published protocol ${ }^{45}$.

542 X-ray data collection

543 Low-noise performance. A large thaumatin crystal (Thau1, $480 \times 240 \times 180 \mu \mathrm{m}^{3}$ ) was

544 measured at $12.4 \mathrm{keV}$ with a flux of $3.5 \times 10^{9} \mathrm{ph} / \mathrm{s}(0.25 \%$ beam transmission $)$. The data sets

545 with full rotation $\left(360^{\circ}\right)$ were measured at $50 \%$ s rotation speed with both JF1M and E1M

546 detectors. The accumulated dose was about $0.6 \mathrm{kGy}$ per data set ${ }^{46}$. The crystal was kept on

547 the goniometer while detectors were exchanged, so both measurements were made with the

548 same position of the crystal and same X-ray beam conditions. The JF1M and E1M detectors

549 were positioned approximately $60 \mathrm{~mm}$ from the sample and operated at $1.136 \mathrm{kHz}$ frame rate

550 and $500 \mathrm{~Hz}$, respectively.

551 Dynamic range study. A thaumatin crystal (Thau2), with a size of $80 \times 80 \times 80 \mu \mathrm{m}^{3}$

552 matching the beam size, was measured at $6 \mathrm{keV}$ with JF1M positioned approximately $40 \mathrm{~mm}$

553 from the crystal. Four $360^{\circ}$ data sets were measured with four settings: (1) $100^{\circ}$ s rotation 
speed and $100 \%$ beam transmission, (2) $50 \%$ s and $50 \%$ transmission, (3) $20 \%$ s and $20 \%$

555 transmission and (4) $1 \%$ and $1 \%$ transmission. Since intensities from (1) and (4) were to be

556 compared, the attenuation factor for (4) was measured with a photodiode and the precise

557 transmission was found to be $1.16 \%$. Therefore, intensities of data with $1 \%$ transmission

558 reported in Fig. 3c and 3d were divided by a factor of 1.16 .

559 Frames were summed: by 2 in case of $50 \%$ transmission, by 5 in case of $20 \%$ transmission

560 and by 100 in case of $1 \%$ transmission. This means all data sets have the same X-ray dose

561 and the same angular increment per summed image. The total dose accumulated through the

562 experiment was estimated at less than $0.5 \mathrm{MGy}$, well below the damaging dose limit for cryo-

563 cooled crystals.

564 Sub-pixel uniformity study. Measurements were carried out with both JF1M and E1M

565 detectors operated with the same frame rate of $1.136 \mathrm{kHz}$ and positioned $45 \mathrm{~mm}$ from the

566 crystal. Two settings were used for the E1M - one with the default 50\% threshold (E1M-50),

567 and the other with $60 \%$ (E1M-60). The integration time of JF1M and E1M was $840 \mu$ s and

$568877 \mu$ s, corresponding to a duty cycle of $95.5 \%$ and $99.7 \%$, respectively. A large thaumatin

569 crystal (Thau3) of about $360 \times 240 \times 240 \mu \mathrm{m}^{3}$ was measured at $6 \mathrm{keV}$ with $15 \%$ beam

570 transmission (flux of $2.5 \times 10^{10} \mathrm{ph} / \mathrm{s}$ ). The same crystal volume was illuminated with the

571 same X-ray beam through the entire experiment.

572 All diffraction data were collected with $10^{\circ} /$ s rotation speed, i.e., step of $0.0088^{\circ}$ in 0.00088

573 second. Five $360^{\circ}$ data sets were measured for each detector. The first two runs were

574 performed with the detector in an initial position, the third one with the detector shifted by 25

$575 \mu \mathrm{m}(1 / 3$ pixel $)$ in both $\mathrm{X}$ and $\mathrm{Y}$ directions from the initial position, the fourth one with the

576 detector shifted by $50 \mu \mathrm{m}(2 / 3$ pixel $)$ in both $\mathrm{X}$ and $\mathrm{Y}$ directions and final one with the detector

577 shifted by $225 \mu \mathrm{m}$ (3 pixel) in both $\mathrm{X}$ and $\mathrm{Y}$ directions the initial position. Only results of the

578 first three experiments are presented. The total dose accumulated through the experiment was 
579 estimated at $1 \mathrm{MGy}$. For data processing images from both detectors were summed by ten, so

580 one frame corresponds to a $0.088^{\circ}$ rotation.

581 Fast native-SAD phasing. For native-SAD at $6 \mathrm{keV}$ X-ray energy, a beam size of $80 \times 80$

$582 \mu \mathrm{m}^{2}$ and the full flux of $2.7 \times 10^{11} \mathrm{ph} / \mathrm{s}$ was used. A thaumatin crystal, with a size matching

583 the beam size (Thau2, $80 \times 80 \times 80 \mathrm{um}^{3}$ ), was measured for a $360^{\circ}$ angular range at $100^{\circ} / \mathrm{s}$

584 rotation speed. The frames with $0.088^{\circ}$ angular increment were used for data processing

585 directly, without summing. The same setup at $6 \mathrm{keV}$ for an aminopeptidase PepN crystal of

$586100 \times 80 \times 80 \mu \mathrm{m}^{3}$ in size was used to measure $720^{\circ}$ angular range at $10^{\circ} / \mathrm{s}$ rotation speed.

587 Ten frames were summed to make one image covering $0.088^{\circ}$ rotation width.

588 For native-SAD at $12.4 \mathrm{keV}$, a lysozyme crystal of $80 \times 80 \times 80 \mu \mathrm{m}^{3}$ in size was used. The

589 lysozyme data set was measured with $100^{\circ}$ s rotation speed and $100 \%$ beam transmission (1.6

$\left.590 \times 10^{12} \mathrm{ph} / \mathrm{s}\right)$

591 X-ray data processing, structure determination and refinement. MX data quality is

592 dependent on phi-slicing ${ }^{12,14}$ and in principle a slower rotation speed allows for finer slicing

593 at a given detector frame rate - which could result in a bias towards slower rotation speeds

594 (up to the point data processing software can correctly account for the extremely weak signal

595 and low background). Therefore prior to data processing, we have performed frame

596 summation to ensure that images obtained at various rotation speeds correspond to a similar

597 rotation angle $\left(0.088^{\circ}\right.$ for $1.136 \mathrm{kHz}$ and $0.100^{\circ}$ for $\left.1.000 \mathrm{kHz}\right)$.

598 Frames were processed with $\mathrm{XDS}^{47}$ software with standard settings. To improve position

599 refinement for the pixel map calculation, the segment refinement feature of XDS was used to

600 account for imprecisions in module positions and the gap size in JF1M and E1M. To allow a

601 direct comparison of intensities for the dynamic range and low-noise performance

602 experiments, scaling factors for integration in XDS were fixed to 1.0. Intensities calculated in 
603 the XDS_ASCII.HKL were divided by the Lorenz-polarization correction factor, to recover

604 the total photon count of a reflection for presentation on Fig. 2c.

605 The calculation of data quality indicators $\left(R_{\text {merge, }} R_{\text {meas }}\right.$ and $\langle I / \sigma>)$ was performed based on

606 XDS and XSCALE outputs using custom python scripts for plotting in finer resolution shells.

607 In the low-noise performance experiment, the normalization of intensity was calculated with

608 the ratio of the duty cycles and the ratio of the absorptions of Si sensor at $2 \theta$ of $32^{\circ}$ (Fig. 2c).

609 The $I / \sigma$ was normalized with the square root of the ratios (Fig. 2d). The duty cycle and sensor

610 thickness are $95 \%$ and $99.7 \%$ and $320 \mu \mathrm{m}$ and $450 \mu \mathrm{m}$ for JF1M and E1M, respectively.

611 Experimental phasing with native-SAD was carried out with SHELXC/D/E ${ }^{30}$ via HKL2MAP

$612 \mathrm{GUI}^{48}$ or with CRANK2 pipeline ${ }^{31}$. The mean peak height for anomalous data $<\mathrm{S}_{\text {anom }}>$ was

613 calculated using ANODE $^{49}$. The structures were refined with phenix.refine ${ }^{50}$ and deposited in

614 the Protein Data Bank (https://www.rcsb.org/).

615 Sub-pixel uniformity characterization. To explore the systematic errors of the detector on

616 the sub-pixel level, we group all the reflections according to where they impinge relative to a

617 pixel center. In this task we benefit from the fact that XDS provides the predicted reflection

618 center to a precision of $1 / 10$ th of a pixel. For each reflection we consider only the fractional

619 part of its position in-pixel units, ignoring its integer part. e.g., if spot is predicted to fall in $\mathrm{x}$ $620=450.1$ pixel and $y=363.5$ pixel, we consider its "in-pixel position" as $x=1, y=5$. Since in

621 XDS the coordinate system in-pixel position $\mathrm{x}=0, \mathrm{y}=0$ corresponds to the center of a pixel,

622 we shift the positions by half pixel to put the origin of the coordinate system at a corner.

623 To quantify such spatial effect, we first calculate deviation from mean for each observation:

$624 \Delta=\sqrt{\frac{n}{n-1}}\left(I_{o b s}-\overline{I_{o b s}}\right)$, 
625 where $\mathrm{n}$ is multiplicity, $I_{o b s}$ is measured intensity and $\overline{I_{o b s}}$ is mean intensity for all symmetry

626 equivalent reflections (including the one in question). The extra term $\sqrt{\frac{n}{n-1}}$ corrects for

627 underestimation of the difference between observation and mean ${ }^{51} . R_{\text {meas }}$ is then simply:

$628 R_{\text {meas }}=\frac{\sum_{h k l} \sum_{n}|\Delta|}{\sum_{h k l} \sum_{n} \bar{T}_{o b s}}$

629 where $n$ is multiplicity. Reflections that were observed only once are ignored in the

630 summation.

631 Next we bin all reflections according to their in-pixel position and for each position $x, y$ we

632 calculate:

$633 \Delta_{x, y}=\sum_{h k l} \sum_{n} \Delta$,

634 where $n$ is the number of reflections that fall into a particular x,y in-pixel position. To allow

635 comparison between in-pixel positions, $\Delta_{x, y}$ can be also normalized in a way similar to R-

636 factors:

$637 \quad \Delta_{x, y}^{\text {norm }}=\frac{\Delta_{x, y}}{\sum_{h k l} \sum_{n} \bar{I}_{o b s}}$.

638 Since $\Delta_{x, y}^{\text {norm }}$ is calculated without taking the absolute value of $\Delta_{x, y}$ before averaging, random

639 differences in intensity measurements should cancel out - a value close to zero of $\Delta_{x, y}^{\text {norm }}$

640 should indicate that there is no systematic error introduced at in-pixel position $\mathrm{x}, \mathrm{y}$. However,

641 if reflections in a particular bin are systematically higher or smaller than the ones in other

642 bins, $\Delta_{x, y}^{\text {norm }}$ should indicate it by a positive or negative value, respectively. $\Delta_{x, y}^{\text {norm }}$ values for

643 each in-pixel position can then be presented on a map which indicates the degree of the non-

644 uniformity across one pixel. The pixel maps calculated with low-resolution reflections $(d>$

$64510 \AA$ ) were presented in Fig. 5a-c.

646 With $\Delta_{x, y}$ in hand one can calculate the effect that charge sharing has on the R-factor value,

647 by calculating the mean of the absolute values of $\Delta_{x, y}$ :

$648 \quad R_{p x l}=\frac{\sum_{x, y}\left|\Delta_{x, y}\right|}{\sum_{h k l} \sum_{n} \bar{I} \overline{o b s}}$, 
649 where $N$ is the number of all reflections with multiplicity of at least two. Due to the fact that $650|a|+|b| \geq|a+b|, R_{\text {meas }}$ is an upper limit for $R_{p x l}$ and comparison of the two values can 651 indicate the share of systematic errors due to sub-pixel non-uniformity in relation to the total 652 uncertainty.

653 For calculations we apply a standard cutoff for reflection intensities I $>-3 \sigma$. Since we are 654 interested in systematic deviations of reflection intensities, we also include misfits, marked in 655 XDS_ASCII.HKL with negative $\sigma$ values, in all statistics calculations presented in Fig. 5d-f $656 \quad\left(R_{p x l}\right.$ and $\left.R_{\text {meas }}\right)$.

657 Photon count-rate estimation. Peak photon rate for a reflection observation was 658 approximated as the following:

$659 \quad$ Rate $=\frac{M A X C}{(0.075 m m)^{2}} \frac{v}{\Delta \phi}$,

660 where MAXC is the highest count observed in a single pixel from a single frame for a 661 particular reflection (column MAXC in INTEGRATE.HKL from XDS), $v$ is the rotation 662 speed in $\%, \Delta \phi$ is the rotation range of a single image in degrees and $0.075 \mathrm{~mm}$ is the pixel 663 pitch. This number is only the lower estimation of the peak rate, since while a crystal rotates, 664 the intensity of a reflection varies according to its rocking curve, especially if $\Delta \phi$ is larger 665 than mosaicity (as in our case). However, if one compares data set collected with the same $666 \Delta \phi$, the incoming photon rate should be comparable in both. The spread in observed values 667 might come from the different spread of counts inside a peak (charge sharing).

668 In Fig. 3d, we present correlations of peak rates of two JF1M data sets collected on the same 669 crystal at $1 \%$ s and $100 \%$ s rotation speed with corresponding beam transmission of $1 \%$ and $670100 \%$ (see above for exact experimental details). To ensure equivalent $\Delta \phi$ frame summation 671 was performed on the slower data set. For the correlation plot, we choose only reflections 672 with identical Miller indices from both data sets, and no symmetry equivalence was applied. 673 In this way, peak rates calculated in $1 \% \mathrm{~s}$ JF1M data, multiplied by 100 , is an approximation 
674 of the "true" rates for $100 \%$ s data. This is then compared with the measured rate values in $675100^{\circ} / \mathrm{s}$ data.

676 For reference, we calculate peak rate values using a theoretical model for a paralyzable

677 counter, where the relation between true count-rate $\mathrm{I}_{0}$ and observed count-rate $\mathrm{I}$ is given as:

$678 I=I_{0} e^{-I_{0} \tau}$,

679 where $\tau$ is an energy dependent sensor dead-time. The $\tau$ value used in Fig. $3 \mathrm{~d}$ was taken as

$680280 \mathrm{~ns}$, which is an experimental value determined for $6 \mathrm{keV}$ photons for the PSI

681 manufactured EIGER ${ }^{52}$.

682

683 Data availability

684 All diffraction data have been deposited in figshare depository and are accessible at

685 https://doi.org/10.6084/m9.figshare.6087368. Diffraction data and refined models for native-

686 SAD structures have been deposited in the Protein Data Bank under PDB identifiers 6G89

687 (thaumatin), 6G8A (lysozyme), and 6G8B (PepN).

688

689 Code availability

690 Custom computer code for pixel map and $R_{p x l}$ calculations are available at

691 https://github.com/fleon-psi/JF_analysis_scripts.

692 
694

695

696

697

698

699

700

701

702

703

704

705

706

707

708

709

710

711

712

713

714

715

716

717

718

719

720

721

722

723

724

725
42 Wojdyla, J. A. et al. DA+ data acquisition and analysis software at the Swiss Light Source macromolecular crystallography beamlines. J Synchrotron Radiat 25, 293303, doi:10.1107/s1600577517014503 (2018).

43 Redford, S. et al. Calibration status and plans for the charge integrating JUNGFRAU pixel detector for SwissFEL. J Instrum 11, C11013-C11013, doi:10.1088/17480221/11/11/c11013 (2016).

44 Bernstein, H. J. \& Hammersley, A. P. in International Tables for Crystallography International Tables for Crystallography Ch. Chapter 2.3, 37-43 (2006).

45 Peng, G. et al. Insight into the remarkable affinity and selectivity of the aminobenzosuberone scaffold for the M1 aminopeptidases family based on structure analysis. Proteins: Structure, Function, and Bioinformatics 85, 1413-1421, doi:10.1002/prot.25301 (2017).

46 Paithankar, K. S., Owen, R. L. \& Garman, E. F. Absorbed dose calculations for macromolecular crystals: improvements to RADDOSE. J Synchrotron Radiat 16, 152-162, doi:10.1107/s0909049508040430 (2009).

47 Kabsch, W. XDS. Acta Cryst D66, 125-132, doi:10.1107/s0907444909047337 (2010).

48 Pape, T. \& Schneider, T. R. HKL2MAP: a graphical user interface for macromolecular phasing withSHELXprograms. Journal of Applied Crystallography 37, 843-844, doi:10.1107/s0021889804018047 (2004).

49 Thorn, A. \& Sheldrick, G. M. ANODE: anomalous and heavy-atom density calculation. Journal of Applied Crystallography 44, 1285-1287, doi:10.1107/s0021889811041768 (2011).

50 Afonine, P. V. et al. Towards automated crystallographic structure refinement with phenix.refine. Acta Cryst D68, 352-367, doi:10.1107/s0907444912001308 (2012).

51 Diederichs, K. \& Karplus, P. A. Improved R-factors for diffraction data analysis in macromolecular crystallography. Nat Struct Biol 4, 269-275 (1997).

52 Johnson, I. et al. Eiger: a single-photon counting x-ray detector. J Instrum 9, C05032C05032, doi:10.1088/1748-0221/9/05/c05032 (2014). 

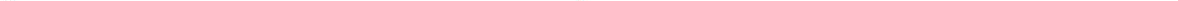
a.

d $(\AA)$

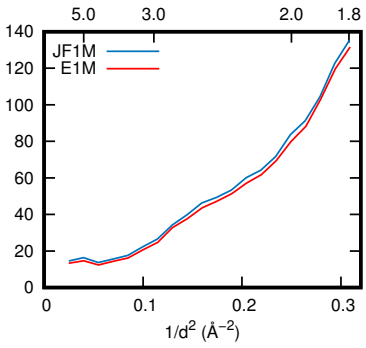

c.

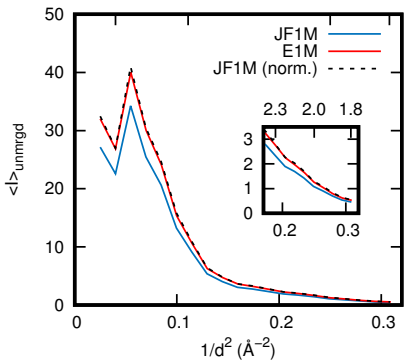

b.

d (Å)

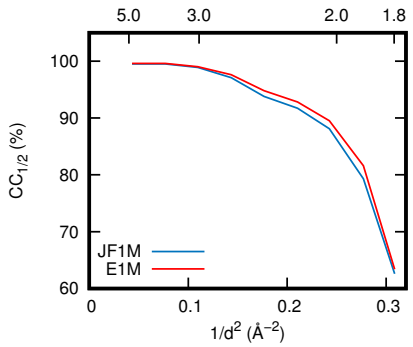

d.

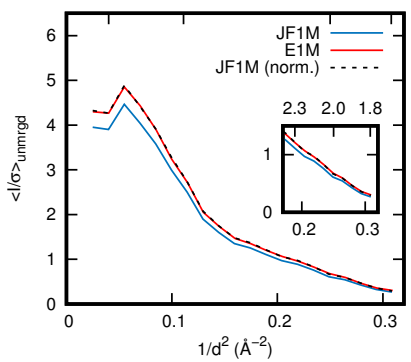




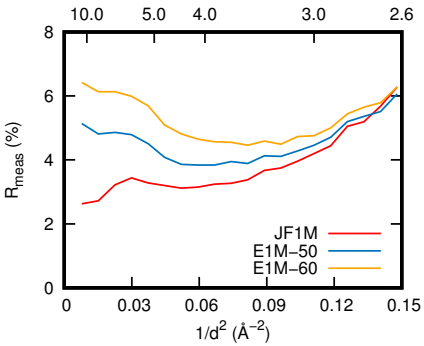

c.
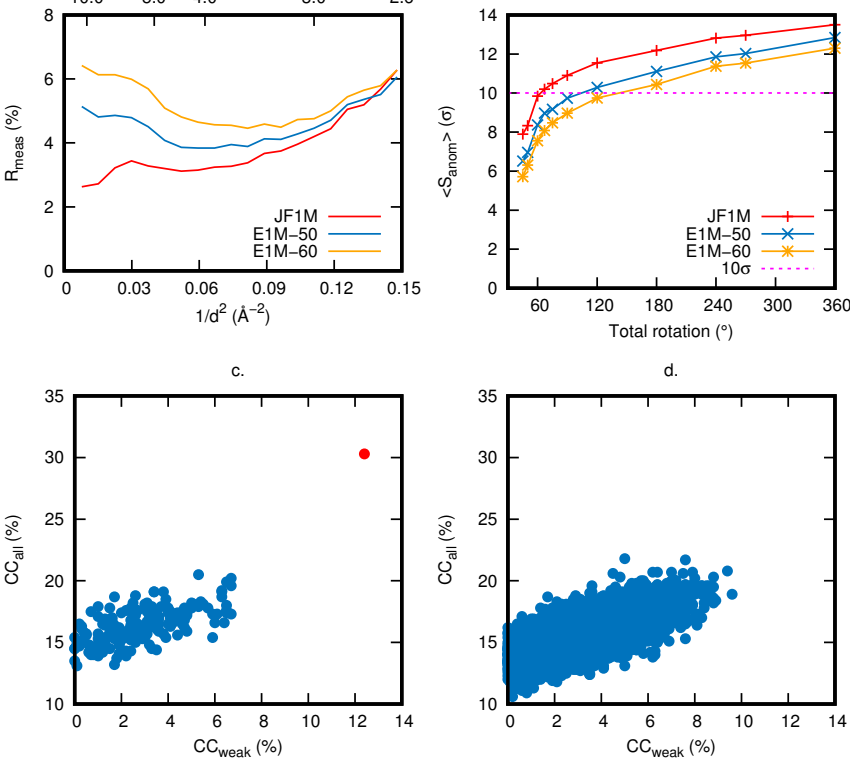

d.

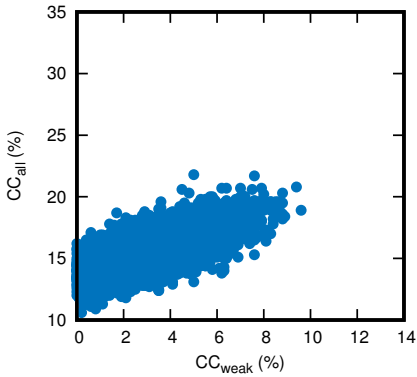


JF1M

a.

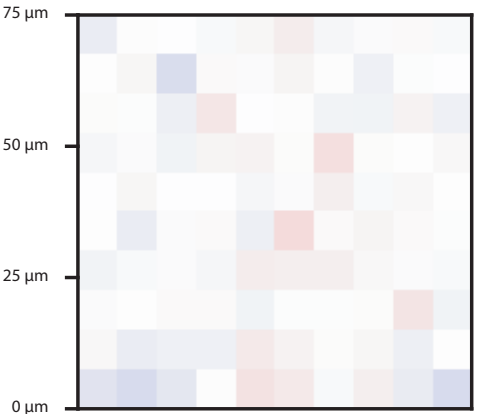

d.

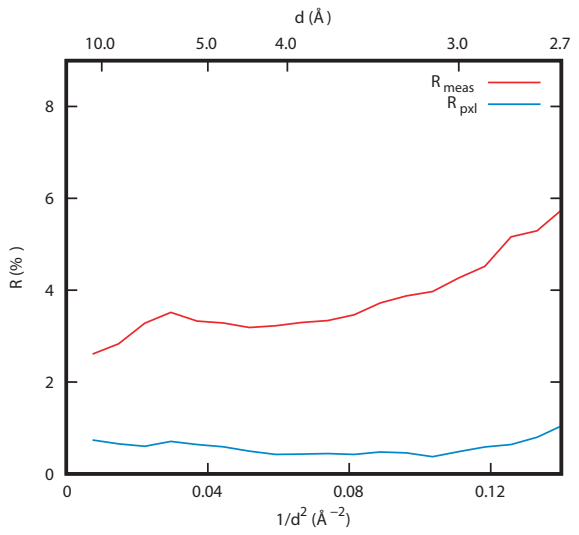

g.

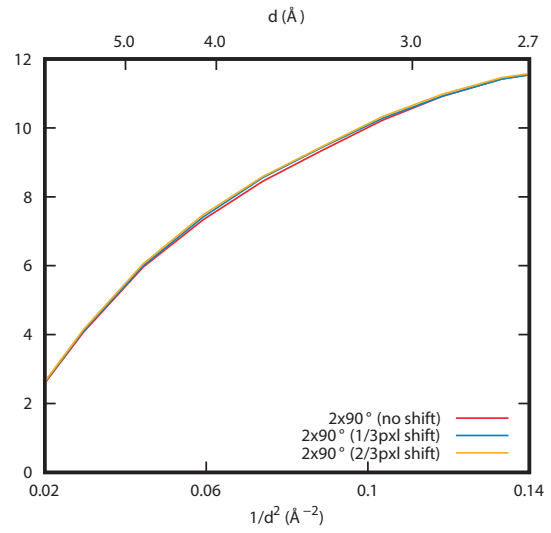

E1M-50

b.

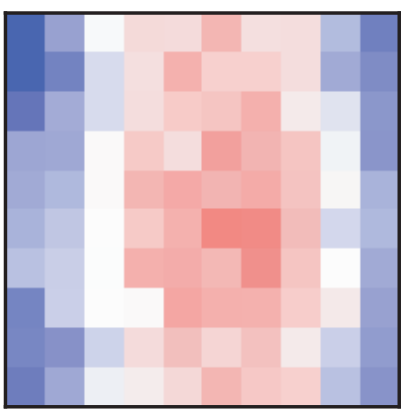

e.

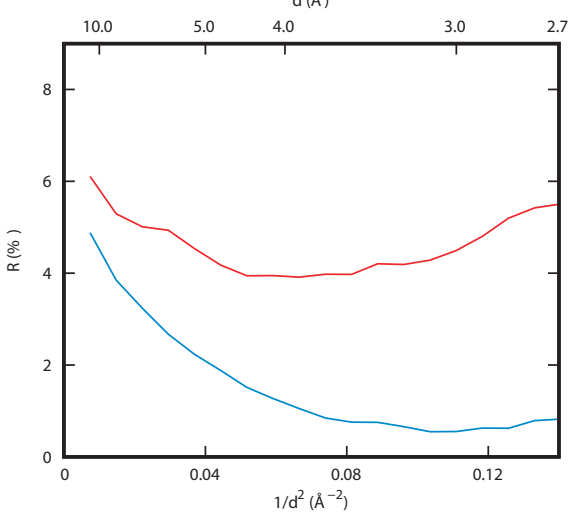

h.

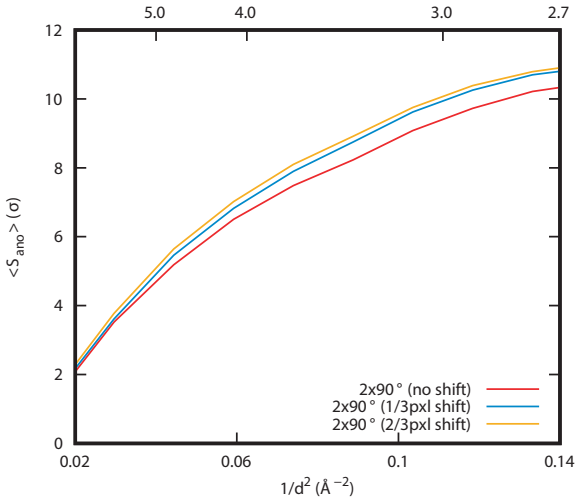

E1M-60

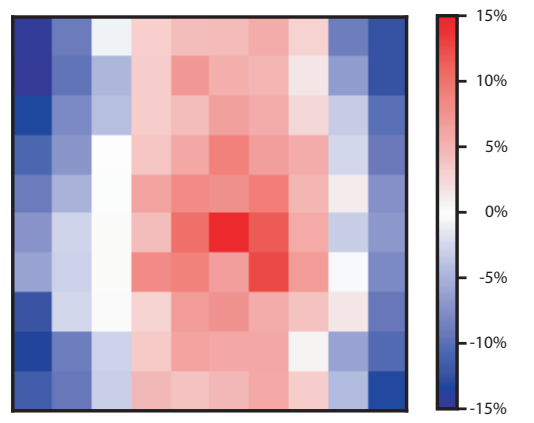

f.
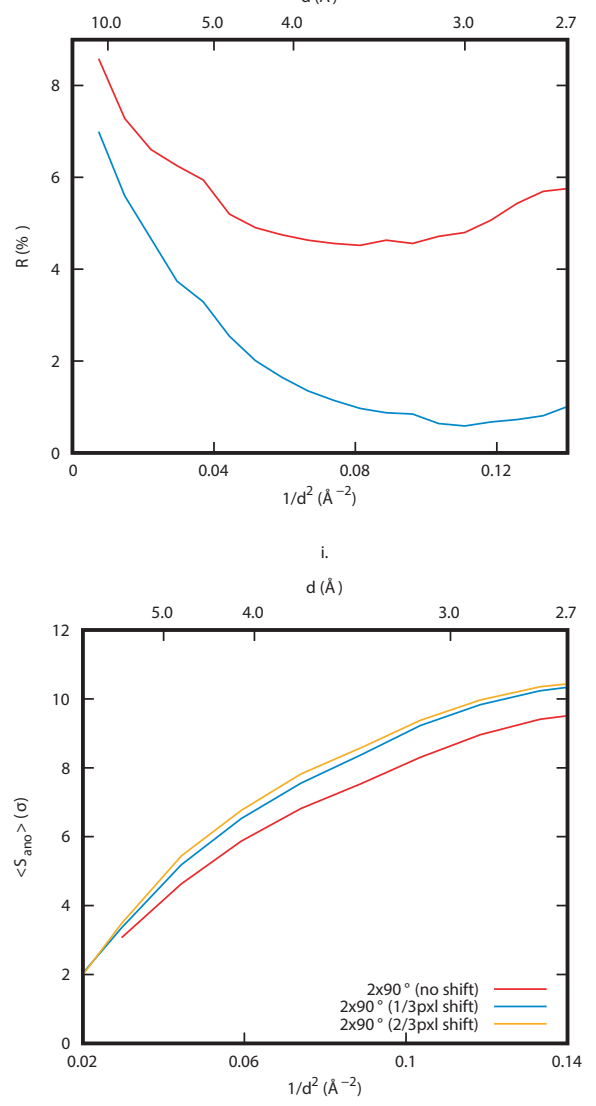
a. Thaumatin ( $6 \mathrm{keV} / 600 \mathrm{~ms} / 60 \mathrm{deg}$.)
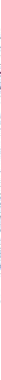

b. Lysozyme (12.4 keV / $5 \mathrm{sec} / 500$ deg.)
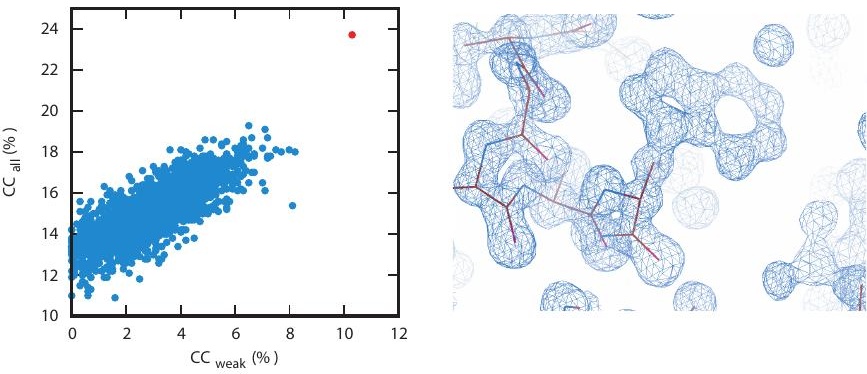

c. Aminopeptidase ( $6 \mathrm{keV} / 60 \mathrm{sec} / 600 \mathrm{deg}$.)
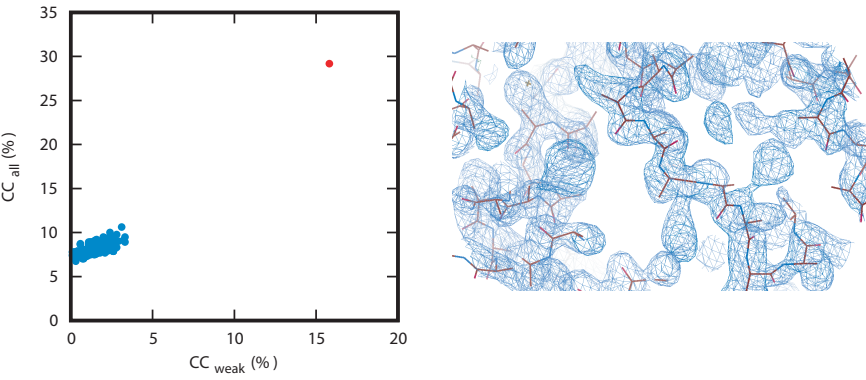